\title{
Characterising the temporal variability of the spatial distribution of animals: an application to seabirds at sea
}

\author{
Grégoire Certain $^{1,2, *}$, Edwige Bellier ${ }^{3,4}$, Benjamin Planque $^{5}$, Vincent Bretagnolle ${ }^{1}$
}

\author{
${ }^{1}$ CEBC-CNRS Chizé UPR 1934, Villiers-en-Bois F-79360 Beauvoir sur Niort, France. \\ ${ }^{2}$ CRELA UMR 6217, Université de La Rochelle, 23 avenue Albert Einstein, 17071 La Rochelle Cedex 9, France \\ ${ }^{3}$ Unité de Biométrie, Avignon, INRA, Domaine Saint Paul, Site Agroparc F-84914 Avignon Cedex 9, France \\ ${ }^{4}$ Centre d'Océanologie de Marseille, Campus de Luminy, Case 901, F-13288, Marseille Cedex 9, France \\ ${ }^{5}$ Département Ecologie et Modèles pour l'Halieutique, Ifremer, B.P. 1105, 44311 Nantes Cedex 03 France.
}

*: Corresponding author : Grégoire Certain, e-mail address : certain@cebc.cnrs.fr

\begin{abstract}
:
Understanding the patterns of spatial and temporal variations in animal abundance is a fundamental question in ecology. Here, we propose a method to quantify temporal variations in animal spatial patterns and to determine the spatial scale at which such temporal variability is expressed. The methodology extends from the approach proposed by Taylor (Taylor, L. R. 1961. Aggregation, variance and the mean. Nature 189: 732-735) and relies on models of the relationship between temporal mean and variance in animal abundance. Repeated observations of the spatial distribution of populations are used to construct spatially explicit models of Taylor's power law. The resulting slope parameters of the Taylor power law provide local measures of the temporal variability in animal abundance. We investigate if the value of the slope varies significantly with spatial location and with spatial scale. The method is applied to seabirds distribution in the Bay of Biscay. We study four taxa (northern gannets, large gulls, auks and kittiwakes) that display distinct geographical distribution, spatial structure and foraging strategy. Our results show that the temporal variability associated to the spatial distribution of northern gannets is high and spatially homogeneous. By contrast, kittiwakes present large geographical areas associated with high and low variability. The temporal variability of auk's spatial distribution is strongly scale-dependent: at fine scale high variability is associated to high abundance, but at large scale high variability is associated to the external border of their distribution range. The method provides satisfactory results and useful information on species spatio-temporal distribution.
\end{abstract}

Keywords: Bay of Biscay, Scale dependence, Seabirds, Spatio-temporal distribution, Taylor's Power Law, Temporal variability. 


\section{INTRODUCTION:}

Explicit consideration of spatial structure have come to play an important role in efforts to understand ecological processes (Fortin \& Dale 2005) and design ecologically sound management and conservation strategies, since most species present highly variable spatial pattern of distribution (Tobin 2004). Furthermore, characterising the temporal variability associated to the spatial distribution of a species is an important prerequisite to the study of the ecosystem functioning and to the establishment of various management, monitoring or restoration programs (see Southall et al. 2006). Together with abundance and variability, a third concept, scale, must be considered to manage population efficiently (Fleisher et al. 1999). The joint measure of both spatial distribution of abundance and temporal variability allows for the distinction between sites with contrasting characteristics: "recurrent sites" correspond to locations where individuals from a particular population are regularly found, "occasional sites" are visited but can be empty at given time whilst "avoided sites" are usually deprived of individuals from the population under study (Bellier et al. 2007). In addition, the spatial extent of these sites must be clearly measured. To this end, measures of spatial structure of densities or occurrences have to be coupled to an index providing a local measure of temporal variability. This index of temporal variability should be built within a scale-dependent framework, in order to identify the spatial scales at which particular types of temporal variability are expressed (e.g. daily, seasonal, interannual). Such information may be critical for conservation or management decisions such as the design of protected areas.

Methods and examples of the measure of temporal variability (or its antonym "temporal stability") exists in the field of soil, hydrology or vegetation sciences (Martinez-Fernandez \& Ceballos 2003, Petrone et al. 2004, Staelens et al. 2006) and in most time follow the methodology developed by Vachaud et al. (1985). This method requires assumptions such as normality or independence between sampling points that can be more easily satisfied in the field of soil science than in the field of ecological surveys of animal populations. Other approaches such as the one proposed by Bjornstadt et al. (1999) characterise explicitly the spatial and temporal scale at which ecological processes such as dispersion are structured, but are not designed to identify the geographic locations where temporal variability is minimal or maximal.

The dispersal patterns of animal populations can alternatively be analysed using Taylor's power law. Taylor's power law (Taylor 1961, Taylor \& Woiwod 1980) is a power function relationship between the variance and mean number of organisms that reflects the spatial heterogeneity of a population within its habitat. If the habitat is divided into a number of equal sized regions, or quadrats, and the number of organisms is enumerated for each quadrat, then the variance $V$ and the mean $M$ number of organisms per quadrat should obey:

$V=b \times M^{a}$

The use of Taylor's power law has been largely discussed, and it provides useful information regarding the dispersal patterns of organisms (reviewed in Kendal 2004). With log-transformed data, the power law becomes a classic linear model with two parameters, the slope $a$ and the intercept $b$. The model can be fitted with either spatial or temporal data (Taylor et al. 1980, Taylor \& Woiwod 1980). Taylor's works highlighted the species-specificity of the slope parameter at a given environmental scale (Taylor \& Woiwod 1982), which was related to intrinsic properties of the species behaviour. Later, Downing (1986) demonstrated that the power law exponent could vary both within a species and be similar between different species. In a spatial context, the slope is considered as an aggregation index. The slope equals unity under the assumption of a random distribution of animals; is lower than unity in the case of regular distribution and greater than unity in the case of aggregated distributions. In a temporal context, the null expectation for Taylor's power law is that the slope of the log variance versus the log mean equals 2 (see Kilpatrick \& Ives 2003 for a demonstration). However, in the field, the slope usually ranges between 1 and 2 (Kilpatrick \& Ives 2003, Kendal 2004). Several mechanisms have been proposed to explain this discrepancy: measurement errors (Titmus 1983), stochastic demography (Anderson et al. 1982) and more recently, negative interactions among species within a community (Kilpatrick \& Ives 2003). Another important property of Taylor's power law is the scale invariance (Kendal 2004, Marquet et al. 2005): whatever the scale at which mean and variance data are calculated, their relationships can always be described by the same power law.

When modelled with temporal data, Taylor's power law indicates that the temporal variability of species abundance increases exponentially with increasing abundance. The strength of this increase reflected by the value of the slope parameter - might or might not change with the spatial location considered. 
Here, we intend to use empirical measures of variations in the slope parameter of Taylor's power law to identify areas of high and low temporal variability, as well as the spatial scale at which these variations are expressed. The main idea is to measure, for a given species, the variations of the slope (estimated with temporal data) as a function of the spatial location and of the spatial scale (see fig. 1 for a schematic representation of the analysis carried out in this study). These results are important to understand how a population is distributed within its habitat, to identify key areas versus highly variables ones and to specify the relevant spatial scale(s). To achieve these goals, we implement the classical Taylor's power law (Taylor 1961, Taylor \& Woiwod 1980) within the context of linear mixedeffect modelling (Pinhero \& Bates 2002) and we treat potential variations of slope in space as a random effect. The proposed methodology is applied to seabird distribution in the Bay of Biscay. Seabirds live in a highly variable environment, with an heterogeneous, patchy and scale-dependent (hierarchical) resource distribution (Ashmole 1971). In turn, the factors affecting their spatial distribution are also scale dependent (Schneider \& Piatt 1986, Russel et al. 1992). At large- and meso-scales, marine top-predator habitats are defined by oceanographic features that control prey concentrations (Russel et al. 1992, Hunt 1997, Croll et al. 1998). Seabirds thus select broad geographical locations for foraging, according to their own experience and to environmental characteristics (Hunt et al. 1999, Bonadonna et al. 2001, Pinaud \& Weimerskirch 2005), but within these large patches, the predictability of predator distribution is low (Fauchald et al. 2002). The processes driving the fine scale distribution of the predators (e.g., prey movements and conspecific behaviour: Wiens 1989, Fauchald \& Erikstaad 2002, Davoren et al. 2002) are highly dynamic and act over short temporal scales. The spatial distribution of seabirds presents therefore complex and scaledependent patterns and is likely to exhibit important temporal variations. For these reasons, seabirds constitute a good model to study the temporal variability of animal distribution. In addition, many seabird species are of environmental concern as they are particularly exposed to the environmental disasters such as oil spill (see Bretagnolle et al. 2004 for an example in the Bay of Biscay) and can serve as indicators of the ecosystem health (Mallory et al. 2006). An accurate description of their spatial distribution, with explicit identification of high and low temporal variability areas is required to identify key areas for their populations and to a larger extent for ecosystem monitoring and management.

\section{MATERIAL AND METHODS:}

\section{Seabird data}

Six aerial surveys were carried on a monthly basis on the continental shelf of the Bay of Biscay (fig. 2), from October 2001 to March 2002. The sampling strategy was designed to fit multiple constraints: the entire shelf area should be covered in a minimal time, and should allow accurate records of every species encountered (see Bretagnolle et al. 2004). The study area was covered in 6-8 days. The high speed of the plane offers the advantage of minimising availability bias (Southwell 1994) which may result from seabird responsive movement (attraction/avoidance). Surveys were carried out only under optimal visibility conditions. A systematic sampling scheme composed of $5000 \mathrm{~km}$ of transects perpendicular to the coast was used to cover the shelf homogeneously (fig. 2). During flights, two observers were constantly watching a $460 \mathrm{~m}$ strip $(2 \star 230 \mathrm{~m}$ each side of the plane). They continuously recorded individual species, numbers, position (provided by GPS) and time of observation. The plane was a six seats lower wing aircraft, and observers took place in the two rear seats to make observations beneath the wings. Environmental data and flight parameters (speed, altitude) were also recorded continuously.

The four most abundant seabird taxa were northern gannets (Sula bassana), large gulls (i.e., herring gulls Larus argentatus for 50\%, lesser black-backed gulls Larus fuscus for $30 \%$, great black-backed gulls Larus marinus for 10\%; and yellow-legged gull Larus michahellis for 10\%), kittiwakes (Rissa tridactyla) and auks (i.e., common murres Uria aalge for $85 \%$, razorbills Alca torda for $15 \%$, and Atlantic puffins Fratercula arctica for 5\%). Statistical analyses were performed at the level of these four taxa. Species were pooled together according to the best identification level obtained from the plane. Among these four groups, two (gannets, kittiwakes) are exclusively composed of one species, one (auks) is mainly composed of one species and only the large gulls present several species with significant proportions of each. A total of 9863 sightings were obtained for these four taxa, adding up to 18981 individuals (see table 1 ). 
Prior to the analysis, transects were converted into a succession of rectangular quadrats (termed "bins") of $5 \mathrm{~km}$ length and $460 \mathrm{~m}$ width. Relative density values (i.e. the number of birds counted within each bin and divided by the bin area, $0.46 * 5=2.3 \mathrm{~km}^{2}$ ) were calculated within every bin for each taxon. Densities are given in 'relative' because it is not possible to ensure that every bird has been counted within each bin. We nonetheless assume that the probability of bird detection was homogeneous during the survey. The choice of $5 \mathrm{~km}$ is a trade off between the proportion of zero values in the data set (statistical distribution of seabird density is inflated with zeros, especially when finer bins are used) and the spatial resolution of the data.

Spatial modelling of bird's distribution.

Before analysing seabird temporal variability, we first attempted to provide detailed maps of their distribution for each month, in order to visualize their variation across months (fig. 1b). These maps were averaged to obtain mean distribution maps, which will be later compared to temporal variability maps. This preliminary goal of visual representation was achieved using the binned data. Monthly maps were calculated only for month containing a sufficient number of individuals. We performed interpolations in two distinct steps that separates large scale patterns from more local spatial structure. In the first step, a deterministic model was used to capture the large-scale component of the seabird spatial distribution, modelled with a Generalised Additive Model (Hastie \& Tibshirani 1990), using a bivariate smooth function of latitude and longitude (see Wood \& Augustin 2002). To avoid overfitting of the data and to restrict density modelling to the large scale habitat, we imposed a penalty during model fitting and constrained the number of knots to a maximum of 8 . The second step consisted in geostatistical interpolation, using ordinary kriging (Cressie 1993), of the spatial residuals obtained from the large-scale model, mainly resulting in fine-scale spatial patterns (local aggregations or gaps). This two-step method is known as the regression-based kriging (Miller et al. 2007). Predicted seabird densities were calculated into a $10 * 10 \mathrm{~km}$ grid. Monthly maps of seabird's relative abundance were obtained by summing large-scale maps (GAM predictions) and residual maps (Geostatistical interpolation) for each month. Then, for each taxon, mean density map were calculated from monthly maps (mean bird density per grid cell). These analyses were achieved with $R$ freeware ( $R$ development core team 2003), using mgcv package (Wood 2001) for GAM modelling and Gstat package (Pebesma \& Wesselin 1998) for geostatistical analysis.

Temporal variability of bird spatial distribution:

We propose to use the slope parameter of the Taylor's power law to measure the variability of animal abundance with time at a given spatial location (fig. 1c). Under the power law, higher slopes constitute an indicator of high temporal variability. The challenge is to be able to detect the potential variations of the slope parameter with space, and then to determine whether these spatial variations are significant. Such a task requires a flexible modelling framework that allows for variations of the model parameters, such as the slope, according to a grouping factor, in our case a spatial block design. In such models, fixed effects are parameters associated to an entire population, while random effects are associated with individual experimental unit drawn at random from a population, resulting in a mixed-effects model (Pinheiro \& Bates 2002). By associating common random effects to observations sharing the same level of a classification factor (spatial location in our case), mixed-effects models flexibly represent the covariance structure induced by the grouping of the data. By associating a random effect to the slope parameter of the Taylor's power law, one can measure the variations of the slope with space. The comparison with a simpler model built without random effects allows for testing if spatial variations of the slope are significant.

To estimate the parameters $a$ and $b$ of Taylor's power law (1) we performed a logarithmic transformation of the data, under which Taylor's Power model becomes:

$\log (V)=a \times \log (M)+B$ where $B=\log (b)$

Mean and variance of animal abundance were obtained using raw data from the 5-km bins (i.e., interpolated data were not used). For each spatial location (bin), the mean and the variance of the bird density was calculated from the six repeated surveys. Our aim is to explore possible variations in space of Taylor's power law parameters. To this end, we divided the Bay of Biscay into blocks. Different block widths were successively used, from $20 \mathrm{~km}$ to $100 \mathrm{~km}$ width, with $5 \mathrm{~km}$ increment. For a given block scale, we obtained i blocks, and within each, j bins. In other words, our blocks correspond to the zones of the figure 1 , and in the same way the bins correspond to the stations. Using the $\mathrm{j}$ bins, a power law could be fitted for each block. The lower block size was set to $20 \mathrm{~km}$ so that each block contains a sufficient number of bins (data) to fit the power law. A block of $20 \mathrm{~km}$ width contains $\mathrm{j}=8$ bins, i.e. 8 mean values and 8 variances. Power laws were only fitted for blocks with $j>=8$ (i.e. when the number of stations is $>=8$ in the fig $1 \mathrm{a}$ ). Our aim is to test if, for a given scale, the slope parameter 
of the power law varies significantly between blocks. In order to account for inter-block variability in model parameters (i.e. power law parameters), a general function is fitted to the data (i.e. all blocks combined) and adjusted for inter-block variability in the model parameters. Linear mixed-effect models are intended for grouped data in which the response can be modelled as a linear function of a set of co-variables (Pinhero \& Bates 2002), which is the case for the present Taylor's power law. Here the data are grouped by blocks representing spatial location, the response variable is the temporal variance and the covariable is the mean. Mixed-effect models allow some parameters to be fixed for all groups (fixed effects) and other to vary between groups (random effects). According to these criteria, two models have been defined and compared for each block scale. The first mixed-effects model is formulated as follows:

$\log \left(V_{i, j}\right)=\bar{A} \times \log \left(M_{i, j}\right)+\bar{B}+b_{i}+\varepsilon_{i, j}$

where $M_{i, j}$ is the mean seabird abundance for the $\mathrm{i}^{\text {th }}$ block and the $\mathrm{j}^{\text {th }}$ bin and $V_{i, j}$ its associated

temporal variance. $\bar{A}$ and $\bar{B}$ are fixed effects corresponding to the conventional parameters (respectively slope and intercept) of Taylor's power law. In addition, one random effect is defined as follows: $b_{i} \sim N\left(0, \sigma_{b}^{2}\right) . b_{i}$ is a random effect for the intercept which correspond to random variations of the intercept values in the $\mathrm{i}^{\text {th }}$ block. The formulation of the mixed-effect model ends by a random error term, $\varepsilon_{i, j} \sim N\left(0, \sigma_{\varepsilon}{ }^{2}\right)$. This first, reduced model (3) is compared to a second one (4) that allows random variations for the slope parameter:

$$
\log \left(V_{i, j}\right)=\left(\bar{A}+a_{i}\right) \times \log \left(M_{i, j}\right)+\bar{B}+b_{i}+\varepsilon_{i, j}
$$

The random effect for the slope $a_{i} \sim N\left(0, \sigma_{a}{ }^{2}\right)$ corresponds to random variations of the slope in the $\mathrm{i}^{\text {th }}$ block.

For each scale used, both models were compared. The comparison was achieved according to the Akaike Information Criterion (AIC), with an ANOVA (see Pinhero \& Bates 2002). Both models were built and compared for each scale, and each time a p-value was extracted indicating whether the second model performed better than the first; i.e., whether slope variations between blocks (i.e. with spatial location) were significant or not. In both models, we assumed a random effect for the intercept parameter, because it has already been observed that this intercept may vary among sampling location and landscape (Taylor et al. 1980). We therefore allowed it to vary each time, to make sure that observed variations of the slope among blocks could not be an artefact due to true variations in the intercept not being stated in the model formulation. The intercept is usually considered as a scaling factor related to sample size (Southwood 1978) and its variations are difficult to interpret in ecological terms (Taylor \& Woiwod 1982).

\section{RESULTS:}

Spatial modelling of bird distribution:

The proportion of deviance explained by the GAMs varied strongly among taxa (table 2). For gannets and kittiwakes, the large scale trends explained $5-15 \%$ of total model deviance (table 2). By contrast it accounted for $16-50 \%$ total deviance in auks and Large Gulls. Ranges of the variogram models varied from $15-50 \mathrm{~km}$, though most were at $20-30 \mathrm{~km}$. No detectable differences could be found between auks, kittiwakes and gannets. Except in February 2002, the variogram models could not be fitted for the Large Gulls. Therefore we used a "nugget" model (i.e. a flat, unstructured variogram), and restricted the interpolation of the gull density at a spatial location to its four nearest neighbours.

Some differences in the level of spatial structuring however appeared among species: the auks and the large gulls had the most significant spatial trends, and the residuals from the GAM were spatially structured for auks, gannets and kittiwakes. Auks presented both an important large scale trend and a high degree of spatial structuring in the GAM residuals, and displayed therefore the strongest spatial structure at any given date.

Monthly maps are constructed by adding the large-scale trends obtained from a GAM and the local spatial structure obtained from kriging interpolation of the residuals of the GAM (see fig. 3 for an illustrated example). Monthly interpolations for all taxa show very high variability of seabird distribution throughout winter (fig. 4). 
The comparison of spatially explicit full model (eq. 4) versus the spatially averaged reduced model (eq. 3) as a function of spatial scale is summarized in fig. 5 which indicates whether both models differs significantly or not for every block size tested. This analysis shows contrasting results for the four taxa considered. For gannets, the full model performed better than the reduced model only at the finest scales (up to $30 \mathrm{~km}$ ). For gulls and kittiwakes, the full model performed better at all tested scales (from 20 to $100 \mathrm{~km}$ ). For auks, the full model performed better cyclically at specific scales (around 40, 60 and $85 \mathrm{~km})$.

We then reported average maps of seabird distribution in winter 2001-2002 (fig. 6a) together with the spatial repartition of slopes value issued from the model (4), at two different scales $(30 \mathrm{~km}$, fig. $6 \mathrm{~b}$ and $60 \mathrm{~km}$, fig. $6 \mathrm{c}$ ). The figure 6 illustrates that the temporal variability associated to seabird distribution varied among taxa and across spatial location and scale. Among taxa, high slopes are reported in almost all spatial location in gannets and large gulls, low slopes in auks, and both high and low slopes, depending on the spatial location, for kittiwakes. This indicates that auks present the most temporally stable spatial patterns among the four taxa studied.

The temporal variability pattern of gannets presents a smooth north-south gradient of slope values, with highest slopes observed in the northern part of the Bay. The gradient is however apparent only at fine scales as at $60 \mathrm{~km}$ scale, the slope values are homogeneous across the study area. We then conclude that this gradient is not sufficiently strong to be considered significant for the northern gannet, and will retain that its spatial distribution is highly variable, can present "core" areas of distribution associated locally to lower temporal variability (see fig. 6a\&b), but presents strong and spatially homogeneous temporal variability at scales higher than $30 \mathrm{~km}$.

In the large gulls, we found that their temporal variability changed with space at any scale (see fig. $5 b$ ). However the joint interpretation of maps of distribution and variability is not straightforward, possibly in relation to the fact that (i) among the four taxa studied, large gulls provided fewest data (table 1) making the fit of Taylor's power law into each block more sensitive to random errors, and (ii) the pooling of several species together makes the pattern revealed hardly interpretable. In the Bay of Biscay, large gulls are mainly distributed in the northeast of the Bay (near Brittany coast, fig. 6a) which also harbours numerous fishing fleets (Léauté 1997). Temporal stability maps suggest that their spatial distribution during winter is highly variable and that they can be encountered punctually in any point of the Bay (fig. $6 \mathrm{b \& c}$ ).

The distribution pattern observed in auks is very well structured, with high abundances into coastal areas, particularly in front of Loire and Gironde estuaries where specific hydrological structures develop as a result of important river runoff (Puillat et al. 2004). The abundance patch in front of Loire is associated with important temporal variability at both scales, whilst the area located in front of the Gironde estuary presents a scale-dependent temporal dynamics, with higher variability (greater slope values) at a scale of $30 \mathrm{~km}$, and lower variability (smaller slope values) at a scale of $60 \mathrm{~km}$ (fig. $6 \mathrm{~b} \& \mathrm{c}$ ). Therefore, a change in the spatial scale considered leads to the reversion of the temporal variability pattern, with fine scale variability of auk's distribution masked at broad scale.

In the case of kittiwakes, the north-south gradient of temporal variability is obvious at small scale and discriminates clearly between the two kittiwakes patches (north-west and central part of the Bay, fig. $6 a \& b)$. The gradient remains significant at a larger scale, but the temporal variability pattern is modified, as only the northern coastal area (near Brittany coasts) is associated to very important temporal variability (fig. 6c).

\section{DISCUSSION}

Methodological comments and perspectives.

The method proposed provides a practical tool for measuring the temporal variability associated with animal's spatial distribution at different scales. The method is based on the spatial and/or temporal variations in the slope parameter of Taylor's power law which are used to characterise the temporal variability of the ecological processes which control animal abundance.

In the present study, a number of methodological choices were made according to the type and the structure of the dataset analysed. We discuss below the possible implications of these choices.

First, grouping of several species into unique taxa should be avoided when possible. In our case, grouping was due to uncertainty in the taxonomic identification of some species (e.g., auks, sub-adult and juvenile plumages in gulls). Two taxa were mono-specific, one was mainly composed of one species and only one, the large gulls, contained several species with probable different ecological characteristics. This implies that the pattern of temporal variability observed for large gulls is a mix of different specific patterns. It does not describe accurately each specific pattern but rather represent a 
global pattern common to the species grouped into the taxa. Hence, ecological interpretation based on the pattern revealed for large gulls must be carried out with caution.

Also, further methodological developments could be needed to better match different ecological questions. For example, the question of temporal scales is also a major concern for conservation (Fleisher et al. 1999) and could be investigated. For our study, we had only six repetitions of the survey, a minimum to compute Taylor's power law trough time. However, with more repetitions and especially with inter-annual surveys, the results could be extended to characterize inter-annual variability, an important topic to provide useful guidelines for management purposes. The question of temporal scale could be explicitly incorporated into our modelling framework by considering temporal blocks as an additional random effect. However, these improvements require long time series to extract relevant information on the specific temporal scales at which animal abundance are stable or variable.

Another point that could be explored is the presence and the structure of spatial auto-correlation in the slopes. Indeed, spatial autocorrelation is a key component of ecological processes (Koenig 1999). In our analysis, we have partially handled this by using geographical blocks at different spatial scales. However, the issue of spatial autocorrelation could be more directly addressed. A geostatistical analysis of the structure of the spatial variance (Cressie 1993) in the slopes could lead to the definition of the shape and size of particular areas (using kriging interpolation), for example hot spots of low temporal variability. However, modelling spatial autocorrelation should be carried out only in the cases where the slopes are truly varying in space. Our results show that for some species, this latter condition depends on the spatial scale of the analysis. Moreover, such development would require numerous data on temporal variability at the smallest possible spatial scales, in order to model the autocorrelation in the slopes (see Fortin \& Dale 2005 for guidelines). In the case presented in this paper, the minimum grain into which slopes are calculated is $20 \mathrm{~km}$ which leaves few possibilities for an accurate definition of areas of interests.

\section{An hypothesis to explain scale-dependent temporal variability patterns.}

Depending on the species and ecosystems considered, the kind of factors influencing their spatial distribution will change. Most seabird species present scale-dependent distribution pattern related to the hierarchical properties of the marine environment (Schneider \& Piatt 1986, Fauchald 1999, Fauchald et al. 2002). The scale at which environmental factors influence bird spatial distribution changes also with the nature of the factor: the broad scale distribution of predators is often related to bio-climatic variables when the fine scale distribution is influenced by prey distribution (Fauchald et al. 2002; Davoren et al. 2003). To explain the scale-dependent pattern of temporal variability observed in animal distribution, we could hypothesize that the spatial scale at which the temporal variability change significantly with space reflect the scale at which the environment influence animal's spatial distribution. Under this hypothesis, if the temporal variability of spatial distribution of a given species is expressed at large scale, this means that the process controlling the spatial distribution of this species is influenced by a large-scale environmental factor.

Inside our study area, changes in temporal variability of gannet's distribution across space have only been detected at the smallest scales. Thus, within the Bay of Biscay, the environmental controls influencing their distribution should mainly be structured at small scale (prey distribution and/or local oceanographic structure such as up-wellings or eddies). The high and spatially homogeneous temporal variability of gannets spatial distribution suggest that their population is highly mobile, with individuals exploring the Bay of Biscay over large distance to find local areas suitable for feeding. It is therefore possible that large-scale environmental control occurs on gannet populations, but at a wider spatial scale than the spatial extent of our study area, since gannets are widely distributed in the north Atlantic.

Auks presented scale-dependent temporal variability patterns. Theoretical considerations about the hierarchical spatial structure of top predators (in which high density patches at fine scales are nested within low density patches at broader scales) have been developed by Fauchald (1999) and empirical descriptions have been provided for auks (Mehlum et al. 1999, Fauchald et al. 2002, Davoren et al. 2003). In addition, diving birds such auks have restricted dispersion abilities and high metabolic costs. They must stay close to their feeding resource (Ainley et al. 2005). Our analysis suggests that at broad scale, auk's densities in coastal areas are more stable than in offshore area. This can correspond to a first level of hierarchical distribution that reveal broad-scale habitat $(60 \mathrm{~km})$. However, at finer scales, much variability is expected in high density areas rather than in intermediate density areas. The observed variability at fine scale (around $30 \mathrm{~km}$ ) can be related to the successive movements of auks between the large scale patches, perhaps in response to prey movements. Fauchald \& Tveraa $(2003,2006)$ developed a method (First Passage Time, FPT) to identify the scale 
of hierarchical patches on the basis of foraging behaviour of predators, collected with individual loggers. It could be useful to compare the scales identified by the analysis of Taylor's power law (based on repeated surveys and populations movements) to those obtained by data acquired at the individual level.

The temporal variability of kittiwakes spatial distribution is characterized by differences between the north-west and the central patches of abundances. These differences are much clear at smaller scale. Kittiwakes are the only species widely distributed in the central part of the bay, a less productive area than the north-western shelf break (Genin 2004) - were gannets are found - or the southern Brittany (Lazure \& Jegou 1998) were large gulls are distributed. Ballance et al. (1997) hypothesised that at sea, the seabird community was structured by two factors, the competition and the energetic constraints, that operate at the opposite ends of a productivity gradient. The competitive species with high energetic requirements would exclude other species from highly productive area. The kittiwake is a small species, less competitive than larger gulls (which often engage aggressive interactions with other birds) or gannets (that can dive and are more efficient at capturing preys). The observed differences between the two kittiwakes patches in term of variability could be explained by the mechanism proposed by Ballance et al. (1997): kittiwakes which try to exploit the most productive areas must compete with other birds and are sometimes excluded, which in turn increase their variability in term of abundance in the area where competition is important. Even if less productive, the central part of the Bay is also less subject to competition, that can reduce the temporal variability of kittiwakes abundance. This hypothesis implies that the effect of competition on the spatial process of kittiwakes distribution is mainly acting at local scales because it is at these scales that the differences between north and central areas in term of temporal variability are the most important.

\section{Discrimination of temporal variability patterns}

For two species (auks and kittiwakes) the discrimination of stable versus variable areas had been made, with respect to spatial scale: for kittiwakes, the central patch of abundances is less subject to small-scale variation in abundance than the northwestern one. For auks, coastal areas and particularly the one in front of Gironde estuary were stable at large scale but were associated to higher temporal variability at small scale. The use spatial pattern identified as variable in time should be avoided for management purpose. In the Bay of Biscay, recurrent areas (i.e; areas associated to important abundance and low temporal variability) for seabirds were mainly identified at broad scale, suggesting that management decision focusing on seabirds population at sea would be more effective and robust if they concern broad-scale habitat. The case of large gulls allows us to warn the reader of applying our method when no sufficient data are available or when several species with different ecological characteristics are pooled together. The case of northern gannets highlights the possibility that a species does not present stable and variable areas at large scale in a given study area. In this case, the information derived from temporal variability maps built during a unique season is limited, because small-scale sectors identified as the most stable during a given season are more likely to exhibit interannual variations than broad scale habitat. The application of our methodology to inter-annual surveys would increase the inference that can be drawn from temporal variability maps.

\section{CONCLUSION:}

Starting from population census and distribution patterns, we described the way seabirds use the space available in the Bay of Biscay and we were able to develop a hypothesis to explain the observed scale-dependent patterns of temporal variability of their spatial distribution. These results provide insights into the fundamental ecology of seabirds and may be used to support applications such as spatial management or conservation. Our work incorporates explicitly spatial effects into Taylor's model fitted with spatio-temporal data. We showed that for a given species the parameters of Taylor's power law can vary with spatial location, which enabled the identification of key areas and their characteristic scale.

Further steps could be achieved by quantifying the relative importance of each environmental factors on the temporal variability of a given species. To this end, joint recording of seabird distribution and environmental data is needed. The local variation of the slope parameter appears smooth and continuous in our application focusing on marine environment. A different picture could emerge from the analysis of terrestrial data where the environmental mosaic is much more discontinuous with clear boundaries (Steele 1989). 


\section{ACKNOWLEDGEMENT:}

We wish to thank the team that managed the survey during the winter 2001-2002, especially Rodolphe Bernard, Thibaut Dieuleveult and Sylvie Houte; thanks also to Michel Métais and the Ligue pour la Protection des Oiseaux who managed the program with the Ministère de l'Ecologie et du Développement Durable. We also thank Julien Reydel for his support during database building, and many thanks to the three anonymous referees who provided very constructive comments.

\section{REFERENCES}

Ainley, D. G. et al. 2005. Physical and biological variables affecting seabird distributions during the upwelling season of the northern California Current. - Deep-Sea Research II 52: 123-143.

Anderson, R. et al. 1982. Variability in the abundance of animal and plant species. - Nature 296: 245248.

Ashmole, N. P. 1971. Seabird ecology and the marine environment. - Avian Biology 1: 223-286.

Ballance, L. T. et al. 1997. Seabird Community structure along a productivity gradient: importance of competition and energetic constraint. - Ecology 78: 1502-1518.

Bellier, E. et al. 2007. Historical fluctuations in spawning site of anchovy (Engraulis encrasicolus) and sardine (Sardina pilchardus) in the Bay of Biscay from 1967 to 2004. - Fisheries Oceanography 16: 115.

Bjornstad, O. et al. 1999. Synchrony and scaling in dynamics of voles and mice in northern Japan. Ecology 80: 622-637.

Bonadonna, F. et al. 2001. Foraging ground fidelity and route-choice tactics of a marine predator: the Antarctic fur seal (Arctocephalus gazella). - Marine Ecology Progress Series 223: 287-297.

Bretagnolle, V. et al. 2004. Distribution maps and minimum abundance estimates for wintering auks in the Bay of Biscay, based on aerial survey. - Aquatic Living Resource 17: 353-360.

Cressie, N. A. C. 1993. Statistics for spatial data. - Wiley inter-science.

Croll, D. A. et al. 1998. An integrated approach to the foraging ecology of marine birds and mammals. - Deep-Sea Research 45: 1353-1371.

Davoren, G. K. et al. 2002. Scale dependent associations of predators and prey: constraints imposed by flightlessness of common murres. - Marine Ecological Progress Series 245: 259-272.

Davoren, G. K. et al. 2003. Distributional patterns of a marine bird and its prey: habitat selection based on prey and conspecific behaviour. - Marine Ecological Progress Series 256: 229-242.

Downing, J. A. 1986. Spatial heterogeneity: evolved behaviour or mathematical artefact? - Nature 323: 255-257.

Fauchald, P. 1999. Foraging in a hierarchical patch system. - The American Naturalist 153: 603-613.

Fauchald, P. and Erikstad, K. E. 2002. Scale dependent predator-prey interactions: the aggregative response of seabirds to prey under variable prey abundance and patchiness. - Marine Ecological Progress Series 231: 279-291.

Fauchald, P. et al. 2002. Seabird and marine oil incident: is it possible to predict the spatial distribution of pelagic seabird? - Journal of Applied Ecology 39: 349-360.

Fauchald, P. and Tveraa, T. 2003. Using first passage time in the analysis of area-restricted search and habitat selection. - Ecology 84: 282-288.

Fauchald, P. and Tveraa, T. 2006. Hierarchical patch dynamics and animal movement patterns. Oecologia 149: 383-395.

Fleisher, S. J. et al. 1999. Sampling in precision IPM: when the objective is a map. - Phytopathology 89: 1112-1118.

Fortin, M. J. and Dale, M. 2005. Spatial Analysis: A Guide for Ecologists. - Cambridge University Press.

Genin, A. 2004. Bio-physical coupling in the formation of zooplankton and fish aggregations over abrupt topographies. - Journal of Marine Systems 50: 3-20.

Hastie, T. J. and Tibshirani, R. J. 1990. Generalised additive models. - Chapman and Hall.

Hunt Jr, G. L. 1997. Physics, zooplancton, and the distribution of least auklets in the Bering Sea - a review. - ICES journal of Marine Science 54: 600-607.

Hunt Jr, G. L. et al. 1999. Physical processes, prey abundance, and the foraging ecology of seabirds. In Adams, N. J. and Slotow, R. H. Proceedings of the 22th International Ornithological Congress , 2040-2056. Durban, BirdLife, South Africa.

Kendal, W. S. 2004. Taylor's ecological power law as a consequence of scale invariant exponential dispersion models. - Ecological Complexity 1: 193-209. 
Kilpatrick, A. M. and Ives, A. R. 2003. Species interactions can explain Taylor's power law for ecological time series. - Nature 422: 65-68.

Koenig, W. D. 1999. Spatial autocorrelation of ecological phenomena. - Trends in Ecology and Evolution 14: 22-26.

Lazure, P. and Jegou, A. M. 1998. 3D Modelling of seasonal evolution of Loire and Gironde plumes on Biscay Bay continental shelf. - Oceanologica Acta 21: 165-177.

Léauté, J. P. 1997. Les flotilles de pêche de l'Union Européenne dans le golfe de Gascogne vues du ciel. - Oceanologica acta 21: 371-381.

Mallory, M. L. et al. 2006. Marine birds as indicators of Arctic ecosystem health: linking the northern ecosystem initiative to long-term studies. - Environmental Monitoring and Assessment 113: 31-48.

Marquet, P. A. et al. 2005. Scaling and power-laws in ecological systems. - The Journal of Experimental Biology 208: 1749-1769.

Martinez-Fernandez, J. and Ceballos, A. 2003. Temporal Stability of Soil Moisture in a Large-Field Experiment in Spain. - Soil Science Society of America Journal 67: 1647-1656.

Mehlum, F. et al. 1999. Scale-dependent correlations between the abundance of Brünnich's guillemots and their prey. - Journal of Animal Ecology 68: 60-72.

Miller, J. et al. 2007. Incorporating spatial dependence in predictive vegetation models. - Ecological Modelling 202: 225-242.

Pebesma, E. J. and Wesseling, C. G. 1998. Gstat: a program for geostatistical modelling, prediction and simulation. - Computer \& Geosciences 24: 17-31.

Petrone, R. M. et al. 2004. Statistical characterization of the spatial variability of soil moisture in a cutover peatland. - Hydrological Processes 18: 41-52.

Pinaud, D. and Weimerskirch, H. 2005. Scale-dependent habitat use in a long-ranging central place predator. - Journal of Animal Ecology 74: 852-853.

Pinheiro, J. C. and Bates, D. M. 2002. Mixed-effects models in S and S-Plus. - Springer.

Puillat, I. et al. 2004. Hydrographical variability on the French continental shelf in the Bay of Biscay, during the 1990s. - Continental Shelf Research 24: 1143-1163.

R Foundation for Statistical Computing. R: A language and environment for statistical computing. [1.8.1]. 2003. R Foundation for Statistical Computing.

Russell, R. W. et al. 1992. Foraging in a fractal environment: Spatial patterns in a marine predatorprey system. - Landscape Ecology 7: 195-209.

Schneider, D. C. and Piatt, J. F. 1986. Scale-dependent correlation of seabirds with schooling fish in a coastal ecosystem. - Marine Ecology Progress Series 32: 237-246.

Southall, E. J. et al. 2006. Seasonal space-use estimates of basking sharks in relation to protection and political-economic zones in the North-east Atlantic. - Biological Conservation 132: 33-39.

Southwell, C. 1994. Evaluation of walked line transect counts for estimating macropod density. Journal of Wildlife Management 58: 348-356.

Southwood, T. R. E. 1978. Ecological methods with particular reference to the study of insect populations. - Chapman et al.

Staelens, J. et al. 2006. Spatial variability and temporal stability of throughfall deposition under beech (Fagus sylvatica L.) in relationship to canopy structure. - Environmental Pollution 142: 254-263.

Steele, J. H. 1989. The ocean "landscape". - Landscape Ecology 3: 185-192.

Taylor, L. R. 1961. Aggregation, variance and the mean. - Nature 189: 732-735.

Taylor, L. R. and Woiwod, I. P. 1980. Temporal stability as a density-dependent species characteristic. - Journal of Animal Ecology 49: 209-224.

Taylor, L. R. et al. 1980. Variance and the large scale spatial stability of aphids, moths and birds. Journal of Animal Ecology 49: 831-854.

Taylor, L. R. and Woiwod, I. P. 1982. Comparative synoptic dynamics. I. Relationships between interand intra-specific spatial and temporal variance/mean population parameters. - Journal of Animal Ecology 51: 879-906.

Titmus, G. 1983. Are animal species really aggregated? - Oikos 40: 64-68.

Tobin, P. C. 2004. Estimation of the spatial autocorrelation function: consequences of sampling dynamic populations in space and time. - Ecography 27: 767-775.

Vachaud, G. et al. 1985. Temporal stability of spatially measured soil water probability density function. - Soil Science Society of America Journal 49: 822-828.

Wiens, J. A. 1989. Spatial scaling in ecology. - Functional Ecology 3: 385-397.

Wood, S. N. 2001. mgcv: GAMs and Generalized Ridge Regression for R. - Rnews 1/2: 20-25.

Wood, S. N. and Augustin, N. H. 2002. GAMs with integrated model selection using penalized regression splines and applications to environmental modelling. - Ecological Modelling 157: 157-177. 
Table 1: Summary statistics of sightings performed between October 2001 and March 2002.

\begin{tabular}{ccccc} 
& gannets & large gulls & auks & kittiwakes \\
\hline \hline number of sightings & 3354 & 499 & 2005 & 3564 \\
number of individuals & 6999 & 1549 & 3279 & 6406 \\
largest sighting & 200 & 110 & 40 & 400 \\
mean group size & 2.08 & 3.1 & 1.64 & 1.79
\end{tabular}


Table 2: Model parameters associated with the monthly interpolation of seabird densities. \% of explained deviance (dev.) and estimated degree of freedom (edf) are given for each GAM (large trend maps). For the geostatistical interpolation of GAMs residuals, the parameters of the variogram are given: Nugget (nug.), Sill and Range (ran., given in km). The type of variogram model is indicated; Spherical (Sph), Gaussian (Gau), Exponential (Exp) or pure Nugget (Nug)

\begin{tabular}{|c|c|c|c|c|c|c|c|}
\hline Species & Date & dev. & edf & nug. & sill & ran. & Mode \\
\hline \multirow{6}{*}{ gannets } & "Oct 2001 & 8.07 & 6.38 & "3.05 & 0.66 & 31.6 & Sph \\
\hline & Nov 2001 & 5.26 & 4.70 & 3.39 & 0.62 & 45.4 & Gau \\
\hline & Dec 2001 & 7.69 & 5.37 & 2.05 & 0.47 & 22.8 & Sph \\
\hline & Jan 2002 & 11.50 & 5.91 & 3.09 & 1.31 & 49.8 & Gau \\
\hline & Feb 2002 & 10.10 & 6.64 & 1.05 & 0.87 & 15.5 & Sph \\
\hline & Mar 2002 & 6.75 & 5.61 & 0.91 & 0.14 & 24.6 & Gau \\
\hline \multirow{6}{*}{ large Gulls } & Oct 2001 & 25.50 & 6.07 & 0.19 & 0.00 & 0.0 & Nug \\
\hline & Nov 2001 & 49.70 & 6.35 & 0.24 & 0.00 & 0.0 & Nug \\
\hline & Dec 2001 & 37.00 & 6.20 & 0.27 & 0.00 & 0.0 & Nug \\
\hline & Jan 2002 & 32.30 & 5.65 & 0.23 & 0.00 & 0.0 & Nug \\
\hline & Feb 2002 & 16.60 & 2.29 & 0.21 & 0.05 & 23.3 & Sph \\
\hline & Mar 2002 & 8.37 & 2.00 & 0.98 & 0.00 & 0.0 & Nug \\
\hline \multirow{5}{*}{ auks } & Nov 2001 & 46.20 & 4.82 & 0.29 & 0.18 & 51.0 & Gau \\
\hline & Dec 2001 & 36.70 & 5.59 & 0.20 & 0.26 & 17.4 & Exp \\
\hline & Jan 2002 & 51.90 & 6.76 & 0.83 & 0.28 & 23.0 & Sph \\
\hline & Feb 2002 & 24.50 & 5.61 & 0.70 & 0.45 & 20.0 & Sph \\
\hline & Mar 2002 & 50.10 & 4.60 & 0.93 & 0.66 & 25.1 & Sph \\
\hline \multirow{4}{*}{ kittiwakes } & Nov 2001 & 13.60 & 5.77 & 1.64 & 0.72 & 37.9 & Sph \\
\hline & Dec 2001 & 8.98 & 5.58 & 0.94 & 0.16 & 32.4 & Gau \\
\hline & Jan 2002 & 8.32 & 6.58 & 1.36 & 1.89 & 35.8 & Sph \\
\hline & Feb 2002 & 16.00 & 6.93 & 1.09 & 1.87 & 29.6 & Gau \\
\hline
\end{tabular}


fig. 1.

a) Consider a studied area with several zones spatially distinct :

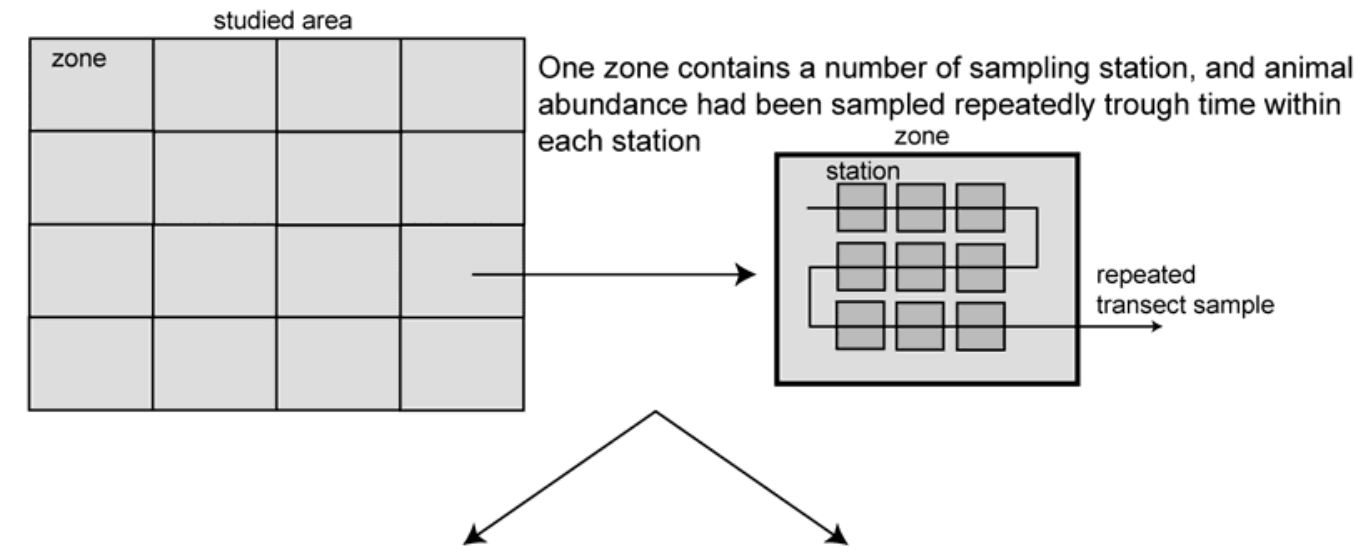

b) Animal abundance is calculated within each zone with classical spatial modelling:

c) From the repeated temporal samples, animal abundance into each station is characterized by a mean and a variance that can be used to model $\downarrow$

GAMs : Large scale components $+$

Geostatistics: Local variations

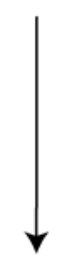
the Taylor's power law for a given zone.

\section{The slope of the Taylor's power law reflect}

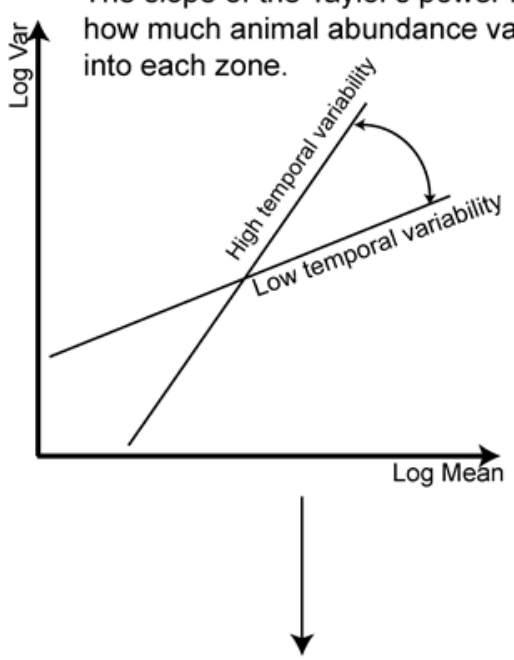

ANIMAL ABUNDANCE MAPS

Measuring the slopes into each zone allows drawing TEMPORAL VARIABILITY MAPS

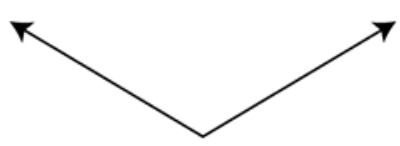

d) Both maps can be compared to identify reccurent, occasional or avoided areas; The effect of the spatial scale of the zones may be investigated

fig. 1. Schematic representation of the analyses carried in the present study. Within a given geographical area, animal abundance is monitored by repeated survey (a). The spatial distribution of animals can be modelled using a combination of Generalized additive models and Geostatistics (b). temporal variability of animal abundance may change with space and with scale. Modelling Taylor's power law for different locations and scales can be used to identify and quantify these variations (c). Mean abundance distribution maps (b) and cartography of Taylor's slopes (c) can be use jointly (d) to identify the spatio-temporal dynamics of marine birds. 
fig. 2.

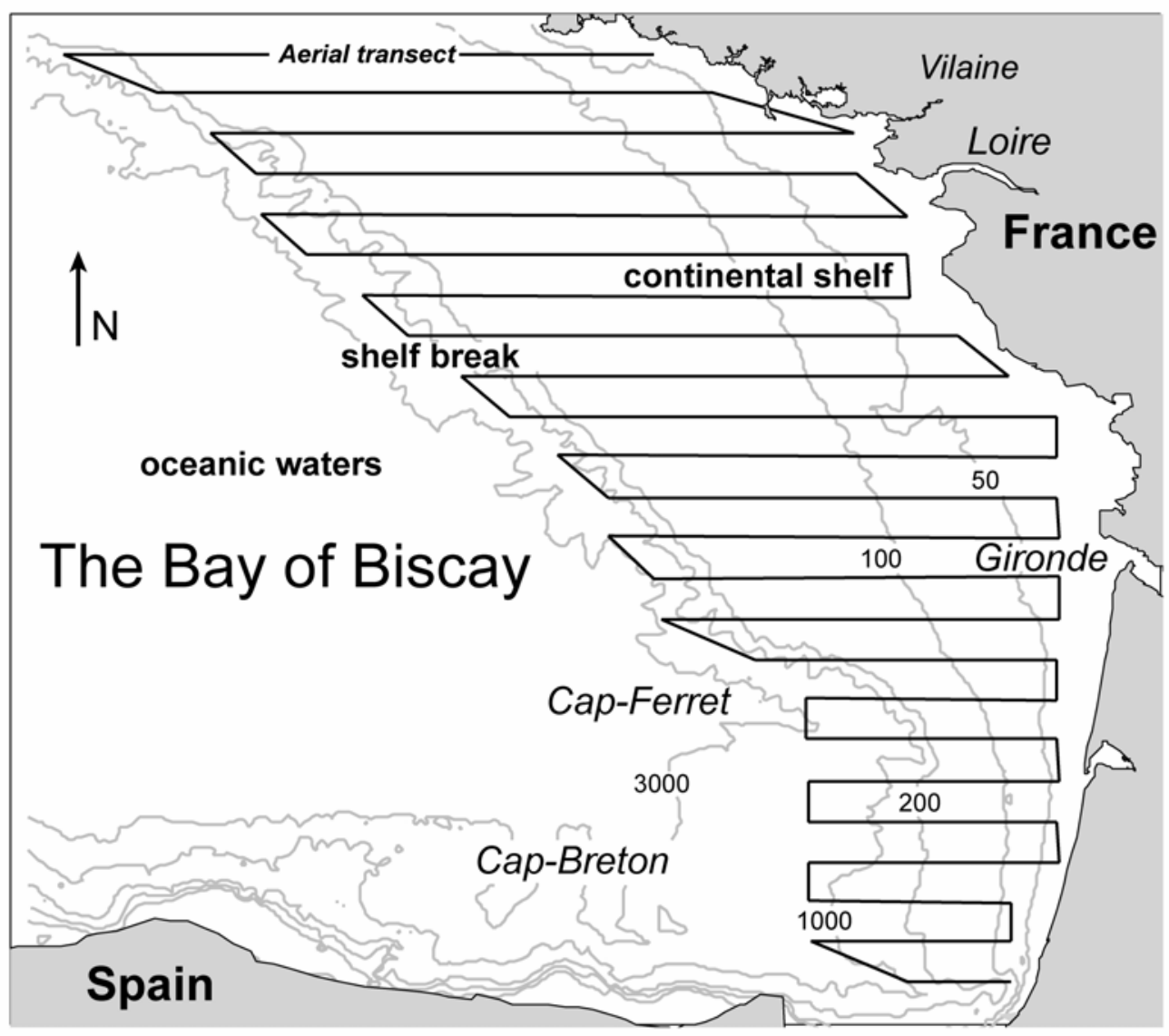

fig. 2. Study area: The Bay of Biscay, $100000 \mathrm{~km}^{2}$ of continental shelf on the French Atlantic coast. The sampling scheme, $5000 \mathrm{~km}$ of transect, composed of 24 transect parallel to the equator over the continental shelf and the upper shelf break. 
fig. 3.
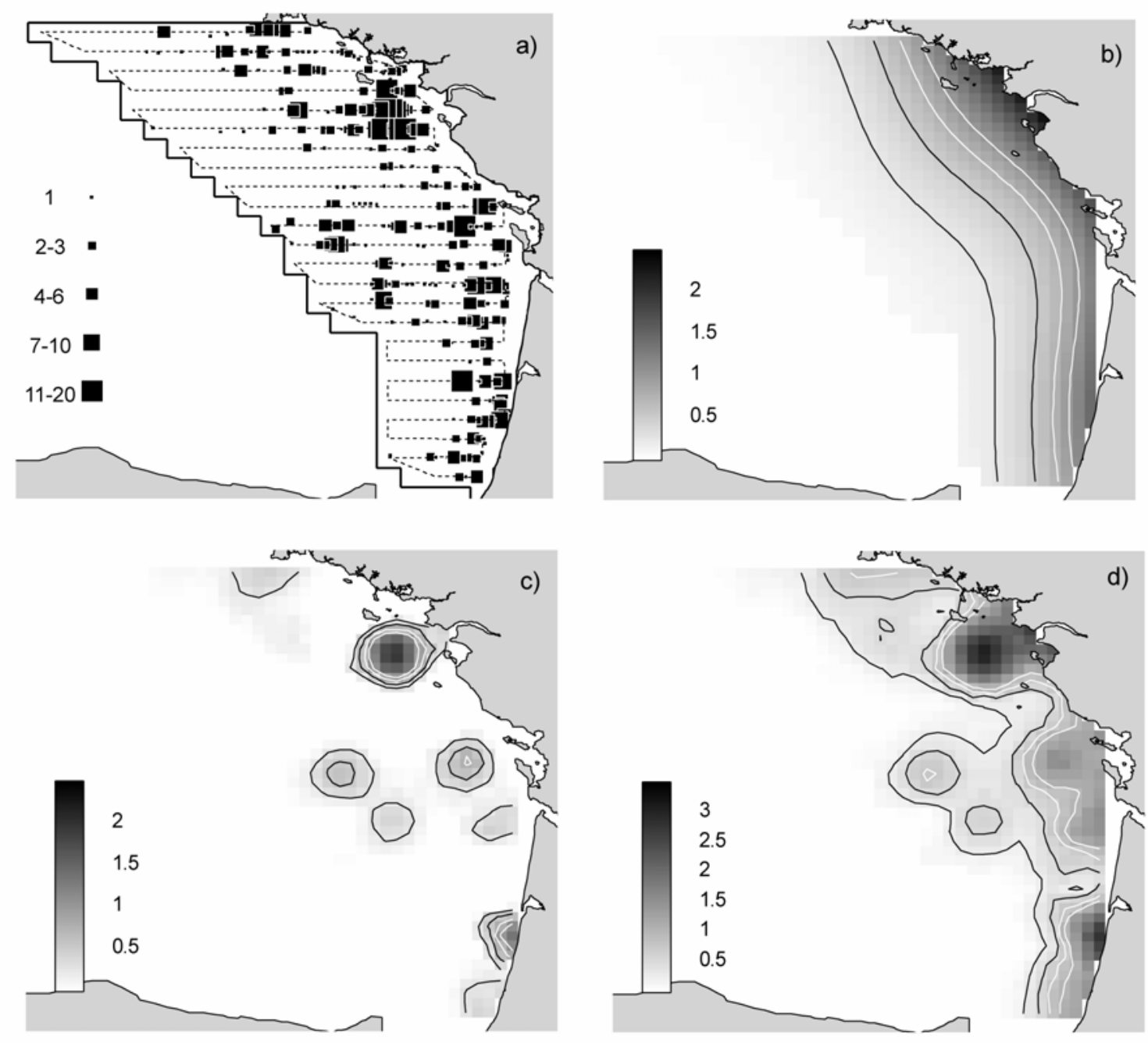

c)

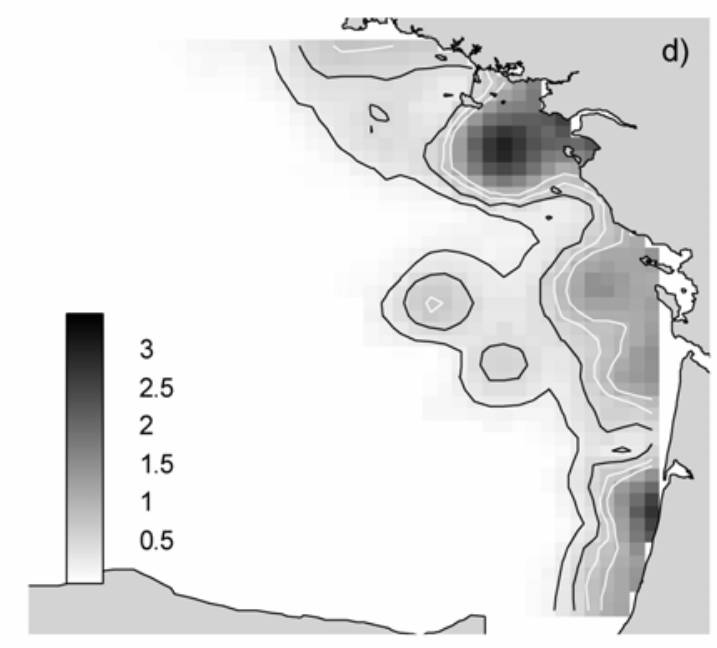

fig. 3. Example of construction of monthly maps for auks in February 2002. a) binned raw data (number of birds/bin). b) large scale pattern (number of birds $/ \mathrm{km}^{2}$ ) modelled with a Generalized Additive Model and c) residual small scale patterns modelled with geostatistics. Large scale distribution (b) and residual small scale patterns (c) are summed to obtain the monthly map (d) of seabirds relative density (number of birds $/ \mathrm{km}^{2}$ ). 
fig. 4.

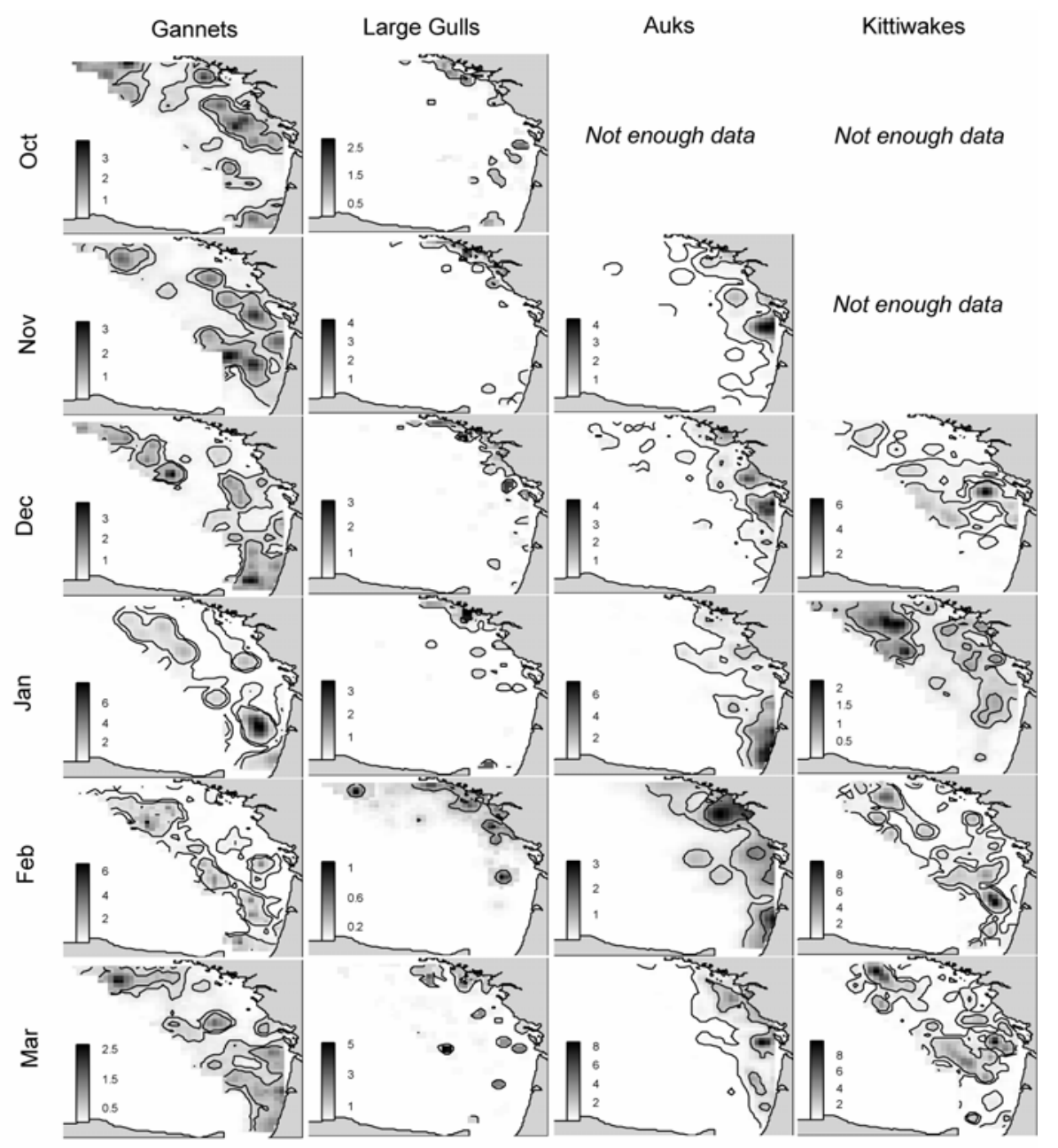

fig. 4. Monthly interpolation of seabird data (relative density expressed in number of birds $/ \mathrm{km}^{2}$ ) following the procedure in fig. 3 for gannets, large gulls, auks and kittiwakes for all cruises (October 2001 to March 2002)

fig. 5 .

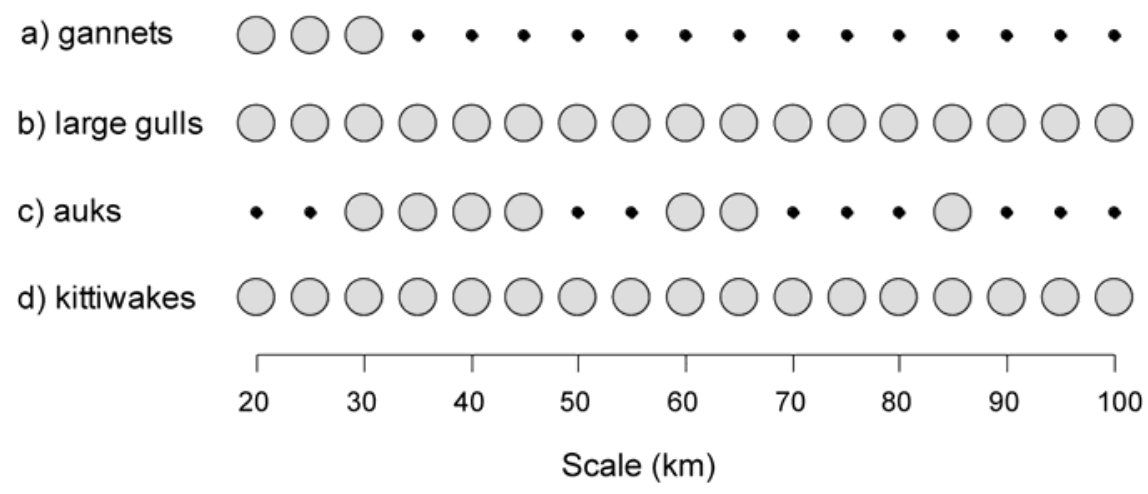

fig. 5. Summary of the comparison between the full model (eq. 3) and the reduced model (eq. 4) as a function of spatial scale ( $x$ axis). Large grey circles indicate that both models differs significantly (i.e. the temporal variability of animal abundance exhibit significant variations with space) and black dots indicates that both models are equivalent (i.e. the temporal variability of animal abundance is homogeneous across the studied area). Results are given for the four taxa studied. 
fig. 6 .
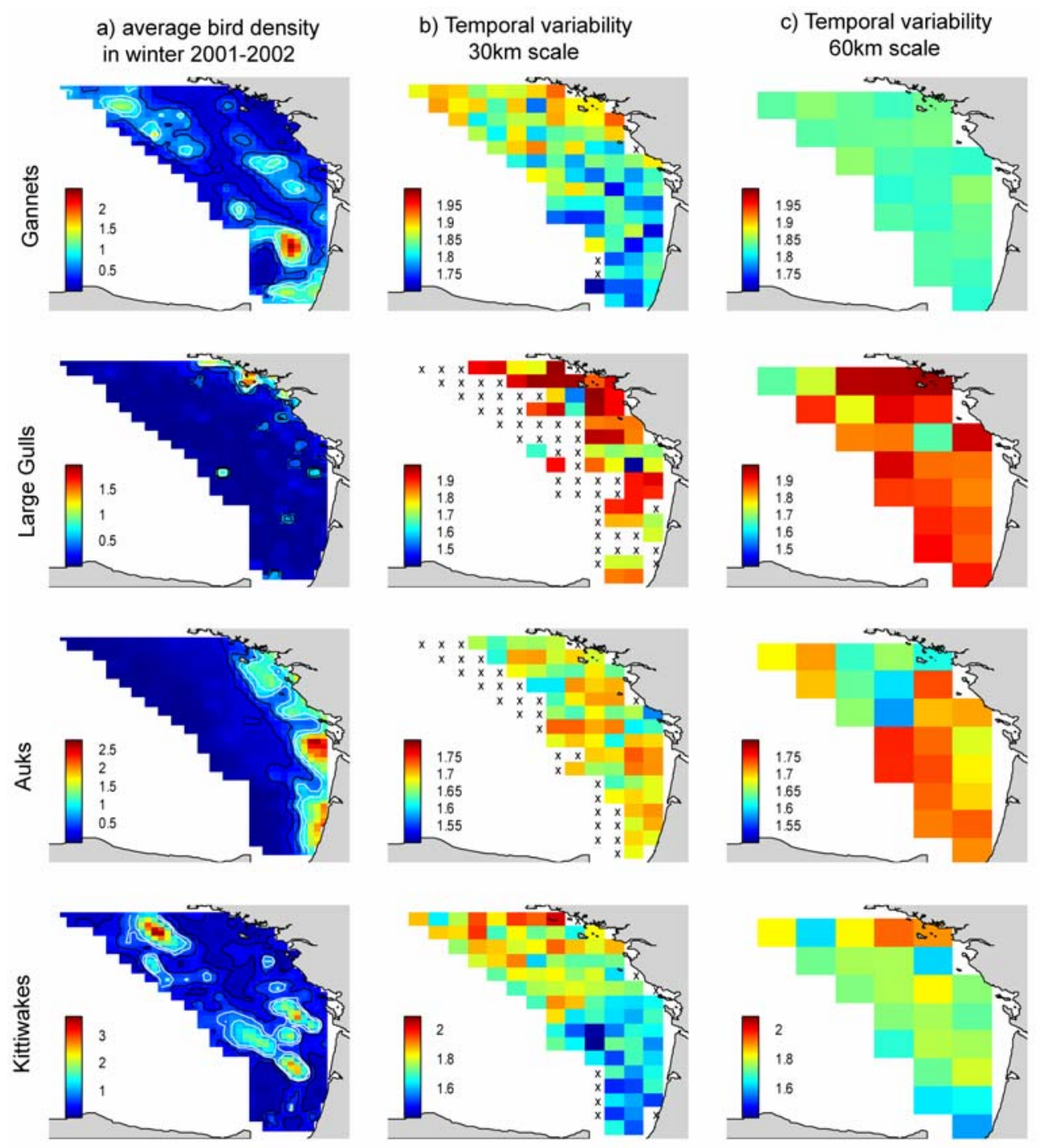

fig. 6. Average seabirds' density (Number of individuals $/ \mathrm{km}^{2}$ ) during winter, calculated by averaging monthly maps (a) ; Spatial distribution of slopes values estimated with the full model (eq. 4) with a block scale of $30 \mathrm{~km}$ (b) and with a block scale of $60 \mathrm{~km}$ (c). Highest slopes reveal important temporal variability. " $x$ " indicates the position of blocs where no sufficient data were available to estimate a slope value for Taylor's power law. 